\begin{abstract}
Objective: Prenatal alcohol exposure (PAE) can cause Fetal Alcohol Spectrum Disorders (FASD) and associated neurodevelopmental impairments. It is uncertain which types of fine motor skills are most likely to be affected following PAE, or which assessment tools are most appropriate to use in FASD diagnostic assessments. This systematic review examined which types of fine motor skills are impaired in children with PAE or FASD; which fine motor assessments are appropriate for FASD diagnosis; and whether fine motor impairments are evident at both 'low' and 'high' PAE levels.

Method: A systematic review of relevant databases was undertaken using key terms. Relevant studies were extracted using a standardized form and methodological quality was rated using a critical appraisal tool.

Results: Twenty-four studies met inclusion criteria. Complex fine motor skills, such as visualmotor integration, were more frequently impaired than basic fine motor skills, such as grip strength. Assessment tools which specifically assessed fine motor skills more consistently identified impairments than those which assessed fine motor skills as part of a generalized neurodevelopmental assessment. Fine motor impairments were associated with 'moderate' to 'high' PAE levels. Few studies reported fine motor skills of children with 'low' PAE levels so the effect of lower PAE levels on fine motor skills remains uncertain.

Conclusion: Comprehensive assessment of a range of fine motor skills in children with PAE is important to ensure an accurate FASD diagnosis and develop appropriate therapeutic interventions for children with PAE-related fine motor impairments.
\end{abstract}


Key Terms: Fetal Alcohol Spectrum Disorders; Motor Skills; Psychomotor Performance; Child Development 


\section{Fine Motor Skills in Children with Prenatal Alcohol Exposure or Fetal Alcohol Spectrum Disorder (FASD)}

Prenatal alcohol exposure (PAE) can result in a range of lifelong neurological impairments in offspring termed Fetal Alcohol Spectrum Disorders (FASD). ${ }^{1-3}$ Diagnoses on the spectrum include Fetal Alcohol Syndrome (FAS), which includes characteristic dysmorphic facial features and growth impairments; partial FAS, with some facial and growth impairments; and alcohol related neurodevelopmental disorder (ARND), in which individuals do not have facial or growth changes. All diagnoses have significant neurological impairments, which can include impaired cognition, executive function, memory, language, attention, social and adaptive skills, and motor skills. $^{2}$

\section{PAE and motor skills}

PAE can affect both fine and gross motor skills. ${ }^{1}$ Early reports noted that infants with FAS had motor impairments ${ }^{4}$, and subsequently orthopaedic and structural defects were also recorded, including clinodactyly (fixed laterally curved fifth finger), camptodactyly (fixed finger flexion), impaired upper limb pronation/supination, tapering of the distal phalanges, and resting and kinetic hand tremors. ${ }^{5-7}$ In animal studies, PAE has been associated with impaired myelination of spinal and peripheral nerves, ${ }^{8,9}$ and impaired motor coordination, response, speed, activity, reflexes, and tone. ${ }^{10,11}$ Neuroimaging studies of individuals with PAE or FASD have identified damage to specific brain regions. Damaged regions which may affect motor skills include the cerebellum, ${ }^{12}$ basal ganglia, ${ }^{13}$ corpus callosum, ${ }^{14}$ and hippocampus. ${ }^{12}$ PAE can also damage neural circuits, including projections which extend into motor and premotor cortices. ${ }^{15}$ There are limited studies of the motor cortex in relation to PAE. However, one study of 
adolescents with PAE concluded that observed motor impairments likely were due to damage to the motor cortex. ${ }^{16}$

\section{Fine motor skills in FASD diagnosis}

Fine motor (FM) skills require the use of small hand muscles and include speed, accuracy, control, coordination, dexterity, visual-motor skills, and eye-hand coordination. ${ }^{17}$ FM skills are important in children because they facilitate increasing independence in self-care tasks such as dressing, eating, brushing hair, and cleaning teeth; academic skills including handwriting, drawing, and using scissors; and participation in play and social activities. ${ }^{18}$ Parents and teachers of primary school aged children with FASD often report they have difficulty with many of these functional tasks. ${ }^{19}$

FASD diagnostic guidelines universally advise that motor skills should be assessed in children with PAE, but variation exists regarding whether FM skills are differentiated from gross motor (GM) skills, and which assessment tools are recommended. The Canadian Guidelines recommend specific tools, such as the Movement Assessment Battery for Children (M-ABC), the Bruininks-Oseretsky Test of Motor Proficiency, and the Beery-Buktenica Developmental Test of Visual-Motor Integration. ${ }^{2}$ The UW 4-Digit Diagnostic Code guidelines do not recommend specific assessment tools, but advise that they should be standardised and validated. ${ }^{20}$ The IOM and CDC guidelines offer no advice about which assessment tools to use. ${ }^{1,3}$

It has been suggested that generalised neurodevelopmental assessments may not detect subtle and specific impairments resulting from PAE. ${ }^{21,22}$ Many neurodevelopmental assessment tools used to assess children with PAE are problematic in terms of FM assessment: some, such as the WISC-IV, ${ }^{23}$ do not include a motor skills component; others, such as the McCarthy Scales of Children's Abilities, ${ }^{24}$ provide an overall motor score which is a composite of FM and GM skills, 
which may mask specific areas of FM or GM impairment; ${ }^{25}$ while others, such as the Griffiths Mental Development Scales, ${ }^{26}$ assess only limited types of FM skills.

\section{FM skills in children with PAE or FASD}

Three systematic reviews have examined the relationship between PAE and motor skills. The first review concluded that only high levels of PAE (10-30 drinks/week) were associated with impaired motor function, although findings were not consistent between studies. ${ }^{27}$ However, this study did not explore whether different types of motor skills were more likely to be impaired, or if FM skills were affected independently of GM skills. A second systematic review and meta-analysis found that GM skills were 2.9 times more likely to be impaired in children aged 0-18 years with 'moderate' to 'high' PAE compared to children without PAE, but this

review did not include FM skills or 'low' PAE $\left(<4\right.$ drinks/week). ${ }^{28}$ A third study of neurological impairments included 13 studies of 'visual and motor' skills and concluded that impairments were not associated with 'mild', 'moderate', or 'binge' PAE, but none of the included assessed

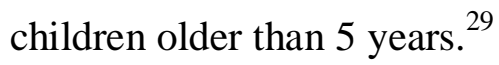

\section{Aims and hypotheses}

Whilst FASD diagnostic guidelines recommend assessing motor skills in children with PAE, it is unclear which types of FM skills are most likely to be impaired or which assessment tools are best to use. It is also uncertain whether impaired FM skills account for the functional difficulties reported by caregivers and teachers of children with FASD. This knowledge is essential to ensure accurate and timely FASD diagnosis, contribute to the development of a FASD neurological profile, employ objective measurement of FM skills over time, and develop therapeutic programs which promote independence by enhancing FM strengths and supporting areas of difficulty. 
This systematic review examined FM skills in primary school aged children (4-12 years) with PAE or FASD, and aimed to:

1. Establish which types of FM skills are impaired following PAE;

2. Identify which FM assessment tools are commonly used with children with PAE or FASD; and

3. Investigate whether different levels of PAE are associated with FM impairments Given the diversity of neural regions affected by PAE, we hypothesised that a range of FM skills would be impaired in children with PAE or FASD, especially those which are more complex and involve multiple neural regions and connectivity. We further hypothesised that FM impairments would be more evident amongst children with high levels of PAE, and that neurodevelopmental assessments which assessed FM skills as part of a generalised assessment battery would not identify FM impairments.

\section{METHODS}

\section{Literature Search}

A search strategy was used to systematically search peer-reviewed journals in the Web of Knowledge, Ovid (Medline, PsychInfo, Maternity and Infant Care, Embase, and Amed), EBSCOhost (CINAHL), ProQuest, SciVerse, The Cochrane Library, Emerald, Informit, OT Seeker, and PEDRO databases. The following key words were combined to identify relevant articles: alcohol*, fetal, maternal, prenatal*, in-utero, and fine motor. No articles were identified which specifically reported FM skills in relation to PAE, so search terms were broadened to include motor, neuro*, development*, sensor*, and visu*. Experts in the field were contacted to identify unpublished studies and reference lists of relevant articles were manually searched.

\section{Inclusion Criteria and Selection Process}


Full texts were obtained for articles which met the study inclusion criteria (Table 1:

Inclusion and exclusion criteria). Included FM skills were: control, precision, speed, or accuracy; VMI or visuomotor precision; in-hand manipulation; hand grasp and release; manual dexterity or coordination; and foundational skills including praxis, grip strength, and finger tapping. Functional FM skills included pencil grasp and writing pressure; handwriting or drawing skills; and adaptive skills with a FM component such as tying shoelaces or doing up buttons. Studies were excluded if they only reported neuromuscular performance such as range of motion, reflexes, or skeletal deformities; GM skills (walking, running, strength, or balance); visual perception (acuity, convergence, tracking, and eye-blink conditioning); visual cognitive skills (visual discrimination, visual memory, form constancy, spatial skills, figure ground, or visual closure); or sensory processing skills.

\section{Data Extraction and Quality Assessment}

There were 24 studies which met all the inclusion criteria. These were summarized by two authors (RD, TJ) using a data extraction form (Table 2: Fine motor assessments and outcomes in children with PAE or FASD; and Supplementary Digital Content Table A: Studies of fine motor skills in children with PAE or FASD). A 10-point critical appraisal tool (CAT) was developed based on STROBE and Health Evidence guidelines to assess the methodological quality and relevance (Table 3: Study quality and relevance). The studies were independently rated by two authors $(\mathrm{RD}, \mathrm{TJ})$ and consensus agreement reached. Studies were classified as having either 'low' (0-4), 'moderate' (5-7), or 'strong' (8-10) methodological quality. Studies which quantified PAE levels as ounces of absolute alcohol/ day or week were converted to drinks/ day or drinks/ week ( $1 \mathrm{oz}$ absolute/ alcohol $=28.35 \mathrm{~g} ; 14 \mathrm{~g}$ approximates 1 standard drink). 
A meta-analysis was not conducted due to the heterogeneity of FM skills and assessment tools, and the variability of PAE levels and FASD diagnoses between studies.

\section{RESULTS}

\section{Literature Search}

We identified 6,259 studies after removal of duplicates (Figure 1: Flow chart for article identification). An additional 10 papers were identified by manually searching reference lists. No unpublished papers were identified from experts in the field. A total of 6,173 papers were excluded because they did not meet inclusion criteria, and a further 72 were excluded for not meeting FM-related criteria. This resulted in 24 studies eligible for inclusion.

\section{Study Characteristics}

Characteristics of the 24 included studies, including PAE levels and FASD daingsoes when provided, are outlined in Supplementary Digital Content Table A - Studies of fine motor skills in children with PAE or FASD. Included studies were published between 1985 and 2012. Sample sizes ranged from 10 to 685 , and included eight small $(\mathrm{n}<50)$, seven medium $(\mathrm{n}=50$ $100)$, and nine large $(\mathrm{n}>100)$ studies.

\section{Risk of Bias and Quality Assessment}

Studies were rated using the CAT (Table 3: Study quality and relevance). Most of the studies had moderate $(58.3 \%)$ or strong $(16.7 \%)$ methodological quality. Half of the studies (50\%) blinded assessors to PAE levels or FASD diagnoses as a strategy to reduce bias. All studies discussed potential confounding factors, but variation existed regarding which confounding factors were considered relevant, and whether these were controlled for statistically. The majority of studies used at least one assessment tool which specifically assessed motor 
skills, as opposed to generalized neurodevelopmental assessments with a FM component (75.0\%), but less than half of the studies assessed more than one type of FM skill (41.7\%).

\section{FASD and PAE}

Most studies included children with FASD diagnoses (70.8\%), but seven of these included only children with the more severe diagnoses of pFAS or FAS (29.2\%). Only three studies reported FM outcomes for children with 'no/low' PAE $<0.14$ oz/ day ( $<2$ drinks/ week); ${ }^{30}$ 'low' PAE (1- 4 drinks/ week); ${ }^{31}$ or 'light/ moderate' PAE (0-1 oz/ day (0-14 drinks/ week). ${ }^{32} \mathrm{~A}$ further three studies included children with 'low' PAE but did not stratify FM outcomes by PAE levels. ${ }^{33-35}$ Thirteen studies only included children with 'moderate' or 'high' PAE, or children with pFAS or FAS (who likely had 'moderate to high' PAE as these diagnoses include the most severe effects of PAE). Not all studies quantified PAE levels, but those that did variously defined 'moderate' and 'high' PAE as: 'moderate'( 5-8 drinks/ week); ${ }^{31}$ 'moderate to high/ heavy' (>10drinks/ week); ${ }^{36}>0.14$ oz/day (or >2 drinks/ week); ${ }^{30}$ 'high/ heavy' (9-14 drinks/ week), ${ }^{31}$ 0.5-1.5 oz/day (or 7-21 drinks/ week) ${ }^{33}>13$ or $>14$ drinks/ week or $>4$ drinks/ occasion; ${ }^{37,38}$ (13.5 oz/day (or 14-49 drinks/ week), ${ }^{32}$ and 'very heavy' ( $>3.5$ oz/ day (or $>49$ drinks/ week). ${ }^{32} \mathrm{~A}$ further four studies included children with a range of FASD diagnoses but did not specify PAE levels. $^{39-42}$

\section{Fine Motor Assessments and Outcomes}

The most common types of FM skills assessed were visual-motor skills and manual dexterity. Researchers used a range of different FM assessment tools (Table 2: Fine motor assessments and outcomes in children with PAE or FASD). 
1. Visual-motor skills $(n=17)$. Studies of visual-motor skills included visual-motor integration (VMI), eye-hand coordination, and visuomotor precision. Assessments included a combination of drawing and functional tasks such as threading beads or posting coins.

\section{(i) Beery-Buktenica Developmental Test of Visual-Motor Integration (Beery VMI)}

$(n=11)$. The Beery $\mathrm{VMI}^{43}$ was the most commonly used assessment in this review. The Beery VMI requires children to copy increasingly complex geometric shapes. All but one study ${ }^{32}$ which used this assessment tool reported significantly impaired VMI skills, including in children with a range of FASD diagnoses. ${ }^{38,39,44,45,46}$ One study identified VMI impairments in children with 'moderate to high' PAE (>10 drinks/ week) in the first trimester; first and second trimesters; and all three trimesters. ${ }^{36}$ In contrast, another study with 5-16 year old children with FAS had impaired VMI skills, but these findings did not extend to children with 'heavy' PAE (unquantified levels) but no FASD diagnosis. ${ }^{47}$ However, a subsequent larger, multi-site study by the same researchers did identify significant VMI impairment in children with 'heavy' PAE (>13 drinks/ week; or $>4$ drinks/ occasion) but no FASD diagnosis. ${ }^{37}$ Only one study did not report VMI impairments when these skills were assessed using the Beery VMI. ${ }^{32}$ This study included 6 year old children with 'none'; 'light to moderate' (0-2 drinks/ day); 'heavy' (2-7 drinks/ day); or 'very heavy' (>7 drinks/day) PAE early in pregnancy. In contrast to other studies, PAE in this study included any alcohol consumption in the year leading up to pregnancy, as the authors considered that this was the most accurate proxy for PAE early in the first trimester.

(ii) Pediatric Environmental Neurobehavioral Test Battery (PENTB): Visual-motor integration test $(n=1)$. The PENTB includes a VMI component, which consists of copying line drawings. ${ }^{48}$ Only one study assessed VMI skills using the PENTB. ${ }^{34}$ When VMI skills were 
analysed using the newly developed 'at-risk alcohol exposure' measure, impairments remained significant after adjustment for potential confounding factors.

(iii) Griffiths Mental Development Scales (GMDS): Eye and hand coordination subscale $(n=3)$. Three studies used the eye and hand coordination subscale of the GMDS, which assesses eye-hand coordination by having the child copy geometric figures, as well as functional tasks such as threading beads. ${ }^{26}$ Two studies found significant eye and hand coordination impairments, including in children with FAS, ${ }^{21}$ and children of alcohol-dependent mothers. ${ }^{49} \mathrm{~A}$ third study of children aged 3-7 years, at least half of whom had 'high' PAE (>15 drinks/ week), identified significant eye-hand coordination impairments. ${ }^{35}$

(iv) A Developmental Neuropsychological Assessment (NEPSY): Visuomotor precision subtest $(n=3)$. The NEPSY visuomotor precision subtest assesses FM speed and accuracy through a timed pencil and paper maze. ${ }^{50}$ One study found no significant visuomotor precision impairments in 5-9 year old children with 'moderate to high' PAE (>10 drinks/ week) in either the first; first and second; or all trimesters. ${ }^{36}$ In contrast, a second study found significant visuomotor precision impairments in 6-12 year old children with FASD. PAE levels were unspecified in this study. ${ }^{42} \mathrm{~A}$ third study used the NEPSY's sensorimotor domain, which includes visuomotor precision, finger tapping, imitating hand positions, and manual motor series subtests, to assess 5-8.5 year old children with FASD, of whom $60 \%$ had 'high' PAE. ${ }^{51}$ This study did not report visuomotor precision findings independently of the other subtests, but found that the overall sensorimotor domain scores were significantly impaired.

2. FM dexterity (speed and coordination) $(n=13)$. FM dexterity was reported in 13 studies using four different assessment tools. 
(i) Pegboard tests $(n=9)$. Pegboard tests assess a range of FM skills, including manual dexterity, precision, speed, and accuracy. Nine studies used pegboard tests and reported varying outcomes. Assessment tools included the Purdue Pegboard Test, the Grooved Pegboard Test, and pegboard tests that comprise part of generalized neurodevelopmental assessment tools, such as the Wisconsin FM Steadiness Battery (WFMSB). Three studies reported non-significant results in children with pFAS or FAS (unspecified PAE levels) ${ }^{52}$ FAS or Fetal Alcohol Effects (a term previously used to describe FASD diagnoses other than FAS) with 'heavy' PAE (unquantified levels); ${ }^{53}$ and children with 'heavy' PAE ( $>14$ drinks/ occasion, or $>14$ drinks/ week). ${ }^{38}$ One study reported non-significant results amongst children with 'none/light' PAE $(<0.14$ oz of absolute alcohol per day, or <2 drinks/ week) compared to children with 'moderate/heavy' PAE ( $>0.14$ oz of absolute alcohol per day, or $>2$ drinks/ week). ${ }^{30}$ Another study reported nonsignificant results in left or right hands in children with FAS born to mothers with alcohol dependency, although conservative $p$ values were used which may have been considered significant in other studies. ${ }^{54}$ At 4 years, children with PAE (7-21 drinks/ week) early in pregnancy, and children who were exposed to an average of more than 1 drink per day throughout pregnancy, took longer to complete the pegboard but did not make more errors than children without PAE. ${ }^{33}$ A different study examined whether different ways of quantifying PAE affected the findings. ${ }^{34}$ This study found that a dichotomous measure of 'at-risk alcohol exposure' was associated with fewer pegs being placed accurately, but these findings were not significant when other measures of PAE were used. A further study reported some significant results when comparing different FASD diagnoses, but findings were not consistent between dominant and non-dominant hands. ${ }^{47}$ In contrast, a larger, multi-site study by the same authors 
found participants with FAS and 'heavy' PAE (>13 drinks/ week; or >4 drinks/ occasion) had impairments in both their dominant and non-dominant hands. ${ }^{37}$

(ii) Movement Assessment Battery for Children (M-ABC): Manual dexterity subarea $(n=2)$. The M-ABC manual dexterity component includes items such as posting coins, threading beads, and completing a paper and pencil drawing trail. ${ }^{55}$ The M-ABC was used in two studies to assess manual dexterity skills and reported contrasting results. The first study found no significant manual dexterity impairments in 5 year olds stratified by different PAE levels. ${ }^{31}$ This study mostly included 'low to moderate' PAE ( $\leq 8$ drinks/ week), with only $1.6 \%(n=11)$ of the sample being exposed to more than 9 drinks per week, and no children being exposed to more than 14 drinks per week. The second study found manual dexterity skills were significantly impaired amongst 7-10 year olds with various FASD diagnoses, as well as children with ADHD. ${ }^{41}$ This study did not report levels of PAE amongst the children with FASD.

(iii) Detroit Tests of Learning Aptitude (DTLA): The timed motor and precision test $(n=1)$. One study ${ }^{39}$ used the DTLA Timed Motor and Precision Test which assesses FM speed and precision by rapidly drawing crosses in circles of decreasing size. ${ }^{56}$ This study found significant differences in FM speed and precision skills amongst children aged 5-18 years with FAS, but not children with FAE. PAE levels were not defined in this study.

(iv) Wisconsin FM Steadiness Battery (WFMSB) $(n=1)$. One study used the FM tests from the WFMSB ${ }^{57}$ to assess 4 year old children with no PAE; children with PAE only in early pregnancy; and children with PAE throughout pregnancy. ${ }^{33}$ This study reported that the children with 'moderate to heavy' PAE (7-21 drinks/ week) early in pregnancy made significantly more errors and were slower to self-correct their errors compared to children with no PAE. These impairments were significant for children with PAE early in pregnancy as well as children with 
PAE (average $\geq 1$ drink/ day) throughout pregnancy. These difficulties remained significant after adjustment for IQ and other confounding factors.

3. Foundational FM skills: grip strength, finger tapping, and praxis.

(i) Grip strength $(n=2)$. Two studies reported grip strength findings with mixed results. The first study identified no significant differences in grip strength amongst 4 year olds with PAE early or throughout pregnancy. ${ }^{33} \mathrm{PAE}$ levels in this study ranged from 0.5 to $1.5 \mathrm{oz}$ of absolute alcohol/ week (7-21 drinks/ week).The second study identified that 5-18 year olds with FAS or FAE had significant impairments when using both their dominant and non-dominant hands. ${ }^{39}$ PAE levels were not defined for the children in this study.

(ii) Finger tapping $(n=3)$. Finger tapping was assessed in three studies with mixed results. Two studies used a mechanical finger tapper to record how many times the child could tap their finger while holding the other fingers stationary, and a third study measured finger tapping as part of the NEPSY sensorimotor domain. One study reported a significant finger tapping impairment in 5-18 year old Native Americans with FAS or FAE in both their dominant and non-dominant hands. ${ }^{39}$ In contrast, the second study found 4 year old children with 'moderate to heavy' PAE in early pregnancy, but not mid-pregnancy, had impaired finger tapping skills. ${ }^{33} \mathrm{~A}$ third study assessed finger tapping skills in 5-8.5 year old children with FASD, of whom $60 \%$ had 'high' PAE (unquantified levels), but finger tapping results were not reported independently of other tasks in the sensorimotor domain. ${ }^{50}$

(iii) Praxis $(n=2)$. One study assessed kinesthetic and dynamic praxis using the 'Imitating Hand Positions' and 'Manual Motor Series' subtests from the Sensorimotor Domain of the NEPSY. ${ }^{50}$ These tests require imitation of static hand positions and rhythmic hand movements. This study found that dynamic praxis, but not kinesthetic praxis, was affected in 5-9 
year old Finnish children with 'moderate to high' PAE ( $\geq 10$ drinks per week). ${ }^{36}$ Dynamic praxis was impaired in children who had been exposed to 'moderate to high' PAE in the first; first and second; and all three trimesters of pregnancy.

(iv) Combined foundational FM skills $(n=2)$. Two studies reported combined outcomes of different types of foundational hand skills. The first study reported outcomes from the NEPSY sensorimotor domain, which includes dynamic and kinesthetic praxis, finger tapping, and visuomotor precision. ${ }^{51}$ These authors found that children with FASD ( $60 \%$ with 'high' levels of PAE) had impaired sensorimotor skills. A second study assessed FM skills in 6-16 year old children with FASD, who had also experienced significant trauma and trauma, using the Pediatric Early Elementary Examination (PEEX-2) or the Pediatric Examination of Educational Readiness at Middle Childhood (PEERAMID-2). ${ }^{40}$ Tasks included lateral preference, imitative finger movements, finger tapping, and sequential finger opposition. These authors did not find identify significant differences between children with or without FASD. PAE levels were not defined in this study.

4. Functional FM skills. Two studies included assessment of functional FM skills as part of a generalized neurodevelopmental assessment and neither found significant impairments. The first study assessed graphomotor skills using the PEEX-2 or the PEERAMID-2 with 6-16 year old children who had experienced trauma, and compared outcomes between children with and without FASD. Tasks included pencil control, pencil speed, and writing the alphabet. ${ }^{40}$ A second study used the 'Draw a Line Slowly' test from The Cincinnati Autonomy Test Battery to assess motor inhibition skills. No impairments were identified in 6 year old children with 'none'; 'light to moderate' (0-2 drinks/ day); 'heavy' (2-7 drinks/ day); or 'very heavy' ( $>7$ drinks/day) levels of PAE early in pregnancy. ${ }^{32}$ 


\section{DISCUSSION}

This review identified 24 studies which assessed FM skills in primary-school aged children with PAE or FASD. A range of FM skills, including visual-motor skills (VMI, visuomotor precision, and eye-hand coordination), manual dexterity (speed, coordination, and precision), foundational FM skills (grip strength, finger tapping, and praxis), and graphomotor (handwriting, drawing, and motor inhibition) skills were assessed using a variety of FM-specific and generalized neurodevelopmental assessments.

Complex FM skills, such as VMI, were consistently impaired in children with 'moderate to high' PAE, and with pFAS or FAS diagnoses. These findings support the rationale that complex skills, which are controlled by several neural regions and involve multiple neural pathways, are more likely to be impaired following PAE. ${ }^{58}$ VMI skills were impaired in most studies which used the Beery VMI, but findings were less consistent when visual-motor skills were assessed using generalized neurodevelopmental assessments such as the PENTB or GMDS. It may be that generalized neurodevelopmental assessments do not assess visual-motor skills in sufficient detail, or alternatively that the constructional visual-motor skills are less sensitive to PAE than graphomotor visual-motor skills. VMI deficits were evident in both young children and adolescents, and also across different trimesters of PAE exposure, making this an important FM skill to assess in children with PAE for both diagnostic and therapeutic purposes.

Pegboard tests were used to assess manual dexterity, precision, speed and coordination, which are also relatively complex FM skills. However, studies which used pegboard tests as a FM outcome measure did not consistently identify impairments, despite most studies only including children with 'moderate to 'high' PAE, who would be most likely to show 
impairments. It is possible that the pegboard tasks may be too simple to adequately detect subtle and complex FM impairments resulting from PAE.

Few studies investigated foundational FM skills, such as grip strength, finger tapping, praxis, and kinaesthesia, and each used different assessment tools, so outcomes were difficult to compare between studies. Only two studies reported grip strength and finger tapping skills, which are relatively basic FM skills, and reported varying results. ${ }^{33,39}$ The contrasting outcomes may be due to age differences of the children in each study, which is consistent with the theory that PAE-related impairments become more pronounced with maturity. ${ }^{59}$ While it is important to assess foundational FM skills in individual children with PAE as they may be affected and therefore warrant therapeutic intervention and support, evidence suggests that they do not provide a reliable marker for FASD presentation and diagnosis.

Only two studies reported functional outcomes of FM skills, and both used assessments of handwriting or drawing as a small part of a generalized neurodevelopmental assessment. ${ }^{32,40}$ One study included children with exposure to trauma, and compared outcomes between those with and without FASD with undefined levels of PAE. ${ }^{40}$ The second study included children with various levels of PAE, which included alcohol consumption in the year prior to pregnancy as a proxy measure for PAE early in pregnancy. ${ }^{32}$ The demographic and methodological differences between these two studies make it difficult to compare outcomes, but neither study identified impaired graphomotor (handwriting or drawing) skills.

Only three studies stratified FM outcomes specific to 'low' PAE, and these studies did not identify FM impairments. 'Low' PAE was variously defined as $<2$ drinks/ week $;{ }^{30} 1$ - 4 drinks/ week; ${ }^{31}$ and 0-14 drinks/ week in the year leading up to pregnancy (as a proxy measure for early first trimester PAE).$^{32}$ Each of these studies reported different types of FM skills (VMI; 
motor inhibition; and manual dexterity), and each used different assessment tools, so outcomes were difficult to compare. The effect of 'low' PAE on specific type of FM skills remains uncertain.

\section{Studies of functional FM skills which did not meet inclusion criteria}

Several other studies of functional FM skills were identified that did not meet inclusion criteria for this review. However, impaired functional FM skills are frequently reported by parents and teachers of children with FASD, and these studies warrant mentioning. Caregivers of 5-8 year old children reported difficulties with adaptive function, many of which require FM skills, including name writing, fastening clothing, and brushing teeth. ${ }^{51}$ One study assessed handwriting abilities in 20 primary school-aged children with FASD and found that they scored well-below average in handwriting legibility, speed, and visuomotor precision. This was an exploratory descriptive study and no control group was used for comparison. ${ }^{60}$

\section{Limitations}

This review had several limitations. Studies had significant methodological differences regarding PAE levels and FASD diagnoses, which hindered the comparability of FM outcomes. Studies were included only if they used standardized assessments commonly available to clinicians. Consequently, novel assessments of FM skills, such as computer and robotic-based studies, were excluded, as were observational assessments, such as finger-nose touching, tremors, and finger localization. Some studies assessed clock and person drawing, and although these tasks require FM skills, they were excluded because outcomes were reported as visualperceptual and visual-spatial skills. ${ }^{49,61}$ More than half of the studies included children older or younger than the target age range of 4-12 years. This reduced certainty that the conclusions are specific to primary school aged children, although FM impairments were still evident in studies 
that included younger children and adolescents. As with all systematic reviews, there is a risk of positive publication bias. This risk was minimized by including studies which reported a range of neurodevelopmental outcomes, not just studies which reported FM skills, and by contacting experts in the field to identify unpublished studies.

\section{Future Directions}

FASD diagnostic guidelines should be updated to advise clinicians that a range of FM skills, particularly those which are more complex, may affected by PAE, and that these should be assessed separately to other motor skills using FM-specific assessment tools. Future studies should clarify what degree of FM impairment should contribute towards a FASD diagnosis. Establishing whether there is a relationship between specific FM skills and functional performance at school and home in children with PAE would be beneficial to those involved in FASD diagnosis, as well as clinicians, caregivers, and teachers of children with PAE.

\section{Conclusion}

This review identified that FM skills are impaired in children with 'moderate to high' PAE, and children with a pFAS or FAS diagnosis. It remains uncertain if 'low' PAE affects FM skills, because few studies reported FM skills in children with PAE, and those that did assessed different types of FM skills and each used different assessment tools. Consistent with our hypothesis, complex FM skills, such as VMI, were more likely to be impaired following PAE than basic FM skills such as grip strength. Further, specific FM assessment tools, such as the Beery VMI, were more likely to detect impairments than generalized neurodevelopmental assessments. However, pegboard tests, although they are a specific FM assessment tool, and manual dexterity is a relatively complex FM skill, did not consistently detect manual dexterity impairments. It is uncertain whether pegboard tests are too basic to detect manual dexterity 
impairments resulting from PAE, or if this type of skill is not affected by PAE. In all likelihood, it is probably a combination of both of these factors. Comprehensive assessment of FM skills in children with PAE or FASD is essential to contribute towards an accurate FASD diagnosis, and inform therapeutic interventions which support specific areas of FM difficulties and enhance individual strengths.

\section{REFERENCES}

1. Centers for Disease Control and Prevention. Fetal Alcohol Spectrum Disorders: Guidelines for referral and diagnosis. 2005. Available from:

www.cdc.gov/ncbddd/fasd/documents/fas_guidelines_accessible.pdf

2. Chudley, AE, Conry, J, Cook, JL, et al. Fetal Alcohol Spectrum Disorder: Canadian guidelines for diagnosis. Can Med Assoc J. 2005;172:1-21.

3. Hoyme, HE, May, PA, Kalberg, WO, et al. A practical clinical approach to diagnosis of Fetal Alcohol Spectrum Disorders: Clarification of the 1996 Institute of Medicine criteria. Pediatrics. 2005;115:39-47.

4. Jones, KL, Smith, DW. Recognition of the Fetal Alcohol Syndrome in early infancy. Lancet, 1973;302:999-1001.

5. Jones, KL, Streissguth, AP. Fetal Alcohol Syndrome and Fetal Alcohol Spectrum Disorders: A brief history. J Psychiat Law. 2010;38:373-382.

6. Marcus, JC. Neurological findings in the Fetal Alcohol Syndrome. Neuropediatrics, 1987;18:158-160. 
7. Smith, DF, Sandor, GG, MacLeod, PM, et al. Intrinsic defects in the Fetal Alcohol Syndrome: Studies on 76 cases from British Columbia and the Yukon Territory. Neurobeh Toxicol and Ter. $1981 ; 3: 145-152$.

8. Chaudhuri, JD. Myelin degeneration in peripheral nerve in chick embryos following continuous ethanol exposure during early gestational period: A preliminary report. Neuroanatomy. 2006;5:50-55.

9. Ramadoss, J, Lunde, ER, Chen, WJA, et al. Temporal vulnerability of fetal cerebellar purkinje cells to chronic binge alcohol exposure: Ovine model. Alcohol Clin Exp Res. 2007:31:17381745.

10. Miller, MW. Exposure to ethanol during gastrulation alters somatosensory-motor cortices and the underlying white matter in the macaque. Cereb Cortex. 2007;17:2961-2971. 11. Schneider, ML, Moore, CF, Becker, EF. Timing of moderate alcohol exposure during pregnancy and neonatal outcome in rhesus monkeys (Macaca mulatta). Alcohol Clin Exp Res. 2001;25:1238-1246.

12. Autti-Rämö, I, Autti, T, Korkman, M, et al. MRI findings in children with school problems who had been exposed prenatally to alcohol. Dev Med Child Neurol. 2002;44:98-106.

13. Mattson, SN, Riley, EP, Sowell, ER, at al. A decrease in the size of the basal ganglia in children with Fetal Alcohol Syndrome. Alcohol Clin Exp Res. 1996;20:1088-1093.

14. Riley, EP, Mattson, SN, Sowell, ER, et al. Abnormalities of the corpus callosum in children prenatally exposed to alcohol. Alcohol Clin Exp Res. 1995;19:1198-1202.

15. Bookstein, FL, Streissguth, AP, Sampson, PD, et al. Corpus callosum shape and neuropsychological deficits in adult males with heavy fetal alcohol exposure. Neuroimage. 2002;15:233-251. 
16. Korkman, M, Kettunen, SS, Autti-Rämö, II. Neurocognitive impairment in early adolescence following prenatal alcohol exposure of varying duration. Child Neuropsychol. 2003;9:117-128.

17. Henderson, A, Pehoski, C. Hand function in the child: Foundations for remediation. 2 nd ed. St. Louis, MO: Mosby Elsevier; 2006.

18. O'Brien, J, Williams, H. Application of motor control/ motor learning to practice. In: CaseSmith J, ed. Occupational therapy for children . 245-273. St. Louis, MO: Elsevier Mosby; 2010. 19. Clarren, SGB. Teaching students with Fetal Alchol Spectrum Disorder; 2004. Available from: www.education.alberta.ca/media/377037/fasd.pdf

20. Astley, SJ, Clarren, SK. Diagnosing the full spectrum of fetal alcohol-exposed individuals: Introducing the 4-digit diagnostic code. Alcohol. 2000;35:400-410.

21. Adnams, C.M, Kodituwakku, PW, Hay, A, et al. Patterns of cognitive-motor development in children with Fetal Alcohol Syndrome from a community in South Africa. Alcohol Clin Exp Res. 2001;25:557-562.

22. O'Leary, CM, Bower, C. Measurement and classification of prenatal alcohol exposure and child outcomes: Time for improvement. Addiction. 2009;104:1275-1276.

23. Prifitera, A, Saklofske, DH, Weiss, LG. WISC-IV clinical assessment and intervention. San Diego, CA: Elsevier Science; 2008.

24. McCarthy, D. Manual for the McCarthy Scales of Children's Abilities. New York, NY: Psychological Corporation; 1972.

25. Larroque, BB, Kaminski, MM. Prenatal alcohol exposure and development at preschool age: Main results of a French study. Alcohol Clin Exp Res. 1998;22:295-303.

26. Griffiths, R. Griffiths Mental Development Scales. Bucks, United Kingdom: ARICD; 1984. 
27. Bay, B, Kesmodel, US. Prenatal alcohol exposure - A systematic review of the effects on child motor function. Acta Obstet Gyn Scan. 2011;90:210-226.

28. Lucas, BR, Latimer, J, Pinto, RZ, et al. Gross motor deficits in children prenatally exposed to alcohol: A meta-analysis. 2014; Manuscript submitted for publication.

29. Flak, AL, Su, S, Bertrand, J, et al. The association of mild, moderate, and binge prenatal alcohol exposure and child neuropsychological outcomes: A meta-analysis. Alcohol Clin Exp Res. 2014;38:214-226.

30. Fried, PA, Watkinson, B. 36- and 48-month neurobehavioral follow-up of children prenatally exposed to marijuana, cigarettes, and alcohol. J Dev Behav Pediatr, 1990;11:49-58.

31. Bay, B, Støvring, H, Wimberley, T, et al. Low to moderate alcohol intake during pregnancy and risk of psychomotor deficits. Alcohol Clin Exp Res. 2010;36:807-814.

32. Russell, M, Czarnecki, DM, Cowan, R, et al. Measures of maternal alcohol use as predictors of development in early childhood. Alcohol Clin Exp Res. 1991;15:991-1000.

33. Barr, HM, Streissguth, AP, Darby, BL, et al. Prenatal exposure to alcohol, caffeine, tobacco, and aspirin: Effects on fine and gross motor performance in 4-year-old children. Dev Psychol. 1990;26:339-348.

34. Chiodo, LM, Janisse, J, Delaney-Black, V, et al. A metric of maternal prenatal risk drinking predicts neurobehavioral outcomes in preschool children. Alcohol Clin Exp Res. 2009;33:634644.

35. Irner, TB, Teasdale, TW, Olofsson, M. Cognitive and social development in preschool children born to women using substances. J Addict Dis. 2012;31:29-44.

36. Korkman, M, Autti-Ramo, I, Koivulehto, H, et al. Neuropsychological effects at early school age of fetal alcohol exposure of varying duration. Child Neuropsychol. 1998;4:199-212. 
37. Mattson, SN, Roesch, SC, Fagerlund, Å, et al. Toward a neurobehavioral profile of Fetal Alcohol Spectrum Disorders. Alcohol Clin Exp Res. 2010;34:1640-1650.

38. Vaurio, L, Riley, EP, Mattson, SN. Neuropsychological comparison of children with heavy prenatal alcohol exposure and an IQ-matched comparison group. J Int Neuropsych Soc. 2011;17:463-473.

39. Conry, J. Neuropsychological deficits in Fetal Alcohol Syndrome and Fetal Alcohol Effects. Alcohol Clin Exp Res. 1990;14:650-655.

40. Henry, J, Sloane, M, Black-Pond, C. Neurobiology and neurodevelopmental impact of childhood traumatic stress and prenatal alcohol exposure. Lang, Speech, Hear Serv. 2007;38:99108.

41. Kooistra, L, Ramage, B, Crawford, S, et al. Can Attention Deficit Hyperactivity Disorder and Fetal Alcohol Spectrum Disorder be differentiated by motor and balance deficits? Hum Movement Sci. 2009;28:529-542.

42. Zhou, D, Lebel, C, Lepage, C, et al. Developmental cortical thinning in Fetal Alcohol Spectrum Disorders. Neuroimage, 2011;58:16-25.

43. Beery, K. E, Beery, NA. The Beery-Buktenica Developmental Test of Visual-Motor Integration. 6th ed. Minneapolis, MN: Pearson Assessments; 2010.

44. Astley, SJ, Olson, HC, Kerns, K, et al. Neuropsyschological and behavioral outcomes from a comprehensive magnetic resonance study of children with Fetal Alcohol Spectrum Disorders. Can J Clin Pharmacol. 2009;16:e178-201.

45. Coles, CD, Platzman, KA, Raskind-Hood, CL et al. A comparison of children affected by prenatal alcohol exposure and Attention Deficit, Hyperactivity Disorder. Alcohol Clin Exp Res. 1997; 21:150-161. 
46. Sowell, ER, Johnson, A, Kan, E, et al. Mapping white matter integrity and neurobehavioral correlates in children with Fetal Alcohol Spectrum Disorders. J Neurosci. 2008;28:1313-1319. 47. Mattson, SN, Riley, EP, Gramling, L, et al. Neuropsychological comparison of alcoholexposed children with or without physical features of Fetal Alcohol Syndrome. Neuropsychology. 1998;12:146-153.

48. Amler, RW, Gibertini, M. Pediatric Environmental Neurobehavioral Test Battery. Atlanta, GA: US Department of Health and Human Services, Public Heath Service, Agency for Toxic Substances and Disease Registry; 1996.

49. Aronson, M, Kyllerman, M, Sabel, KG, et al. Children of alcoholic mothers. Developmental, perceptual and behavioural characteristics as compared to matched controls. Acta Paediatr Scand. 1985;74:27-35.

50. Korkman, M, Kirk, U, Kemp, S. NEPSY: A developmental neuropsychological assessment manual. 2nd ed. San Antonio, TX: Psychological Corporation; 2007.

51. Jirikowic, T, Olson, HC, Kartin, D. Sensory processing, school performance, and adaptive behavior of young school-age children with Fetal Alcohol Spectrum Disorders. Phys Occup Ther Pedi. 2008;28:117-136.

52. Aragón, AS, Kalberg, WO, Buckley, DD, et al. Neuropsychological study of FASD in a sample of American Indian children: Processing simple versus complex information. Alcohol Clin Exp Res. 2008;32:2136-2148.

53. Laforce, R Jr, Hayward, S, Cox, LV. Impaired skill learning in children with heavy prenatal alcohol exposure. J Int Neuropsych Soc. 2001;7:112-114.

54. Janzen, LA, Nanson, JL, Block, GW. Neuropsychological evaluation of preschoolers with Fetal Alcohol Syndrome. Neurotoxicol Terato. 1995;17: 273-279. 
55. Henderson, SE, Sugden, DA. Movement Assessment Battery for Children: Manual. London, United Kingdom: The Psychological Corporation; 1992.

56. Baker, HJ, Leland, B. Detroit Tests of Learning Aptitude. Indianapolis, IN: Bobbs-Merrill Co; 1959.

57. Matthews, CG, Klove, H. Wisconsin Motor Steadiness Battery: Administration manual for child neuropsychology battery. Madison: University of Wisconsin Medical School, Neuropsychology Laboratory; 1978.

58. Kodituwakku, PW. Defining the behavioral phenotype in children with Fetal Alcohol Spectrum Disorders: A review. Neurosci Biobehav Rev. 2007;31:192-201.

59. Astley, SJ. Profile of the first 1,400 patients receiving diagnostic evaluations for Fetal Alcohol Spectrum Disorder at the Washington State Fetal Alcohol Syndrome Diagnostic and Prevention Network. Can J Clin Pharmacol. 2010;17 e132-e164.

60. Duval-White, CJ, Jirikowic, T, Rios, D, et al. Functional handwriting performance in schoolage children with Fetal Alcohol Spectrum Disorders. Am J Occup Ther. 2013;67:534-542.

61. Uecker, A, Nadel, L. Spatial locations gone awry: Object and spatial memory deficits in children with Fetal Alcohol Syndrome. Neuropsychologia. 1996;34:209-223.

Supplemental Digital Content 1 . Table A - Studies of fine motor skills in children with PAE or FASD

Figure 1 legend: Figure 1. Flowchart for article inclusion 


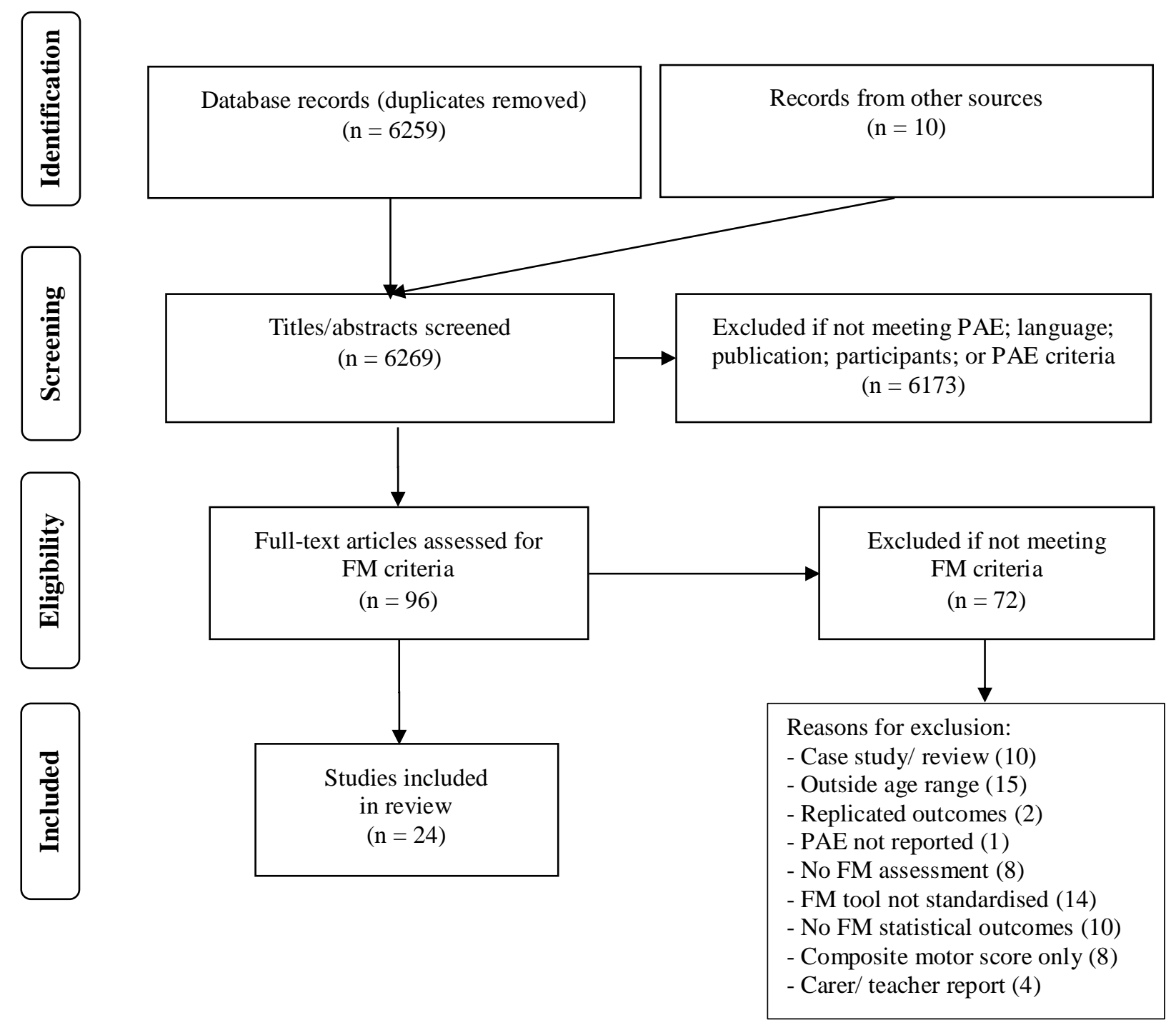

Figure 1. Flowchart for article inclusion 